July 2005

\title{
The Appearance, Apparent Speed, and Removal of Optical Effects for Relativistically Moving Objects
}

Robert J. Deissler

Cleveland State University, r.deissler@csuohio.edu

Follow this and additional works at: https://engagedscholarship.csuohio.edu/sciphysics_facpub

Part of the Physics Commons

How does access to this work benefit you? Let us know!

\section{Original Citation}

Deissler, Robert J. "The Appearance, Apparent Speed, and Removal of Optical Effects for Relativistically Moving Objects." American Journal of Physics 73 (2005): 663-669.

\section{Repository Citation}

Deissler, Robert J., "The Appearance, Apparent Speed, and Removal of Optical Effects for Relativistically Moving Objects" (2005). Physics Faculty Publications. 150.

https://engagedscholarship.csuohio.edu/sciphysics_facpub/150

This Article is brought to you for free and open access by the Physics Department at EngagedScholarship@CSU. It has been accepted for inclusion in Physics Faculty Publications by an authorized administrator of EngagedScholarship@CSU. For more information, please contact library.es@csuohio.edu. 


\title{
The appearance, apparent speed, and removal of optical effects for relativistically moving objects
}

\author{
Robert J. Deissler ${ }^{a}$ ) \\ Physics Department, Cleveland State University, Cleveland, Ohio 44115
}

(Received 6 September 2004; accepted 14 January 2005)

\begin{abstract}
Because various parts of an object are different distances from an observer, and light takes a finite time to reach the observer, the appearance of a relativistically moving object will be very different from that given by the Lorentz contraction. We derive equations that can be applied to a photographic image so that the Lorentz contraction can still be observed. We also give equations that quantify the apparent deformation of the object and plots that show the apparent speed of the object as a function of time. In particular, as an object approaches, its apparent speed can be much greater than the speed of light. In addition, we derive equations that can be applied to photographic images to show how a relativistically moving image will appear. This transformation is applied to photographic images that demonstrate the changes in appearance of a relativistically moving object. The dominate effect is that of appearing to be rotated, that is, the Terrell effect. (C) 2005 American
\end{abstract} Association of Physics Teachers.

[DOI: $10.1119 / 1.1866101]$

\section{INTRODUCTION}

When teaching the subject of special relativity, it is important to stress that measurement and observation are the appropriate words to use in describing length contraction and time dilation. For example, we observe after careful measurement that the length is contracted by the factor of $1 / \gamma$ $=\sqrt{1-v^{2} / c^{2}}$ in the direction of motion for a moving object in comparison to the length in the rest frame of the object. It would be incorrect to state that we "see" the length contraction or that the length "appears" to be contracted by the factor $1 / \gamma$. As first pointed out by Lampa, ${ }^{1}$ and later by Penrose, ${ }^{2}$ Terrell, ${ }^{3}$ and Weisskopf, ${ }^{4}$ what one "sees" and how an object "appears" are very different from what is given by the Lorentz contraction. The reason is that various parts of the object are different distances from the observer, and in order for the light rays from the various parts to arrive at the observer at the same time, they must have left the object at different times. In addition, there is another problem with using the word "appears" in describing the Lorentz contraction and time dilation, even if these optical effects did not occur. What makes Einstein's theory of special relativity so profound is the fact that the length really does contract and time really does slow down. It is not simply a matter of appearances.

If it were possible to take a photograph of a relativistically moving object with a camera that used, instead of photons, particles that travel much faster than the speed of light, then these optical effects would be eliminated and the film would show the object shortened by a factor of $1 / \gamma$ in the direction of motion. However, because such a camera does not exist, we can ask how to correct for the optical effects so that only the relativistic effects will be observed on a photograph taken by an ordinary camera. In this paper we refer to optical effects as those that result from the fact that light takes a finite time to reach the observer and relativistic effects as those that result from the special theory of relativity (that is, the Lorentz transformations). We note that Doppler and intensity effects ${ }^{4}$ also would be present on the film and that these could also be removed, although in this paper we are mainly concerned with geometrical effects.
One of the purposes of this paper is to derive the transformation equations that can be applied to the image on the film (that is, the projection plane) and used to eliminate the optical effects. It is assumed that the object is moving at constant velocity and that the camera can be approximated by a pinhole camera.

Although there have been many papers about the apparent shape of relativistically moving objects, ${ }^{2-17}$ there appears to be no discussion about the apparent speed of relativistically moving objects other than a brief mention. ${ }^{18}$ Because it takes a finite time for light from an object to reach the observer, the apparent position $x_{a}$ of the object will be behind the actual position $x$. Therefore, the apparent speed of the object, $v_{a}=d x_{a} / d t$, will differ from the actual speed, $v=d x / d t$. In particular, we will show that if an object moves past an observer at relativistic speeds, a plot of the apparent angle that the object makes with respect to some reference angle as a function of time is sufficient to determine both the speed of the object and the distance of closest approach. The determination of both these quantities for a relativistically moving object is in contrast to what occurs for an object moving at a nonrelativistic speed, where plotting the angle as a function of time can at most determine the ratio of these two quantities, because changing both the speed and the distance by the same factor will result in the same angle as a function of time.

Although the two-dimensional projection of a relativistically moving object onto a two-dimensional plane has been derived, ${ }^{5}$ equations have not been derived that directly transform a two-dimensional projection of an object in its rest frame to the two-dimensional projection of the apparent coordinates of the object. In this paper we derive this transformation and apply it to some photographic images. In particular, we show that for a small subtended solid angle, the transformation gives the results derived by Terrell. ${ }^{3}$ We also derive equations that quantify the changes in appearance of a relativistically moving object.

In recent years, considerable work has been done on the realistic visualization of relativistically moving objects, ${ }^{13-17}$ including the transformation of photographs from the point 


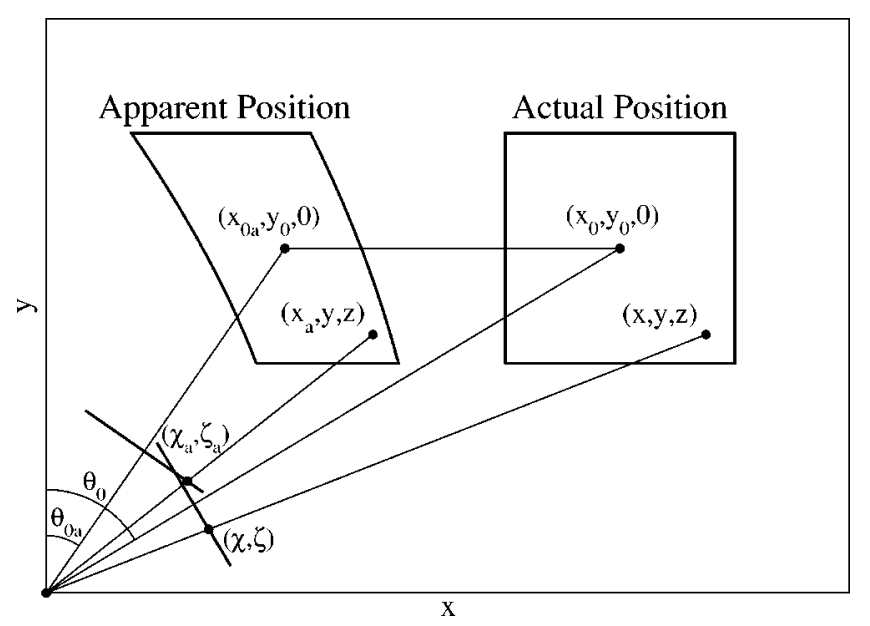

Fig. 1. The actual and apparent position of an object and the twodimensional projection planes.

of view of a moving observer ${ }^{16}$ and from the point of view of a stationary observer. ${ }^{17}$ The novel feature of the transformation of photographs in this paper is the derivation of the transformation from the rest-frame two-dimensional (2D) projection directly to the apparent $2 \mathrm{D}$ projection. These equations let us easily program the transformation and apply it to various photographs.

The transformation of the moving image in the photographs in this paper is from the point of view of the stationary observer, with the image moving past the observer. Because only the image is moving, the transformation is applied only to the moving image, which is then superimposed on the appropriate background. Therefore, the background may be used as a point of reference which aids our understanding of the changes in appearance of the moving object. This feature of including the background is not seen in previous papers in which only the moving object is shown. ${ }^{17}$ We note that Doppler effects and changes in intensity, which are included in previous work, ${ }^{4,13-16}$ are not included here, although these effects could be added to the computer program. ${ }^{19}$

Before continuing with our discussion of the physical appearance of objects, it may be instructive to note how the time appears to change. ${ }^{20}$ If the time between ticks on a clock at rest is $\Delta t^{\prime}$, then the time between ticks on the clock moving at speed $v$ will be measured as $\gamma \Delta t^{\prime}$. However, the time between ticks that will actually be seen is given by the relativistic Doppler shift. For example, if the clock is moving directly toward the observer, the time between ticks will be seen as $\gamma(1-v / c) \Delta t^{\prime}$, and if the clock is moving directly away from the observer, the time between ticks will be seen as $\gamma(1+v / c) \Delta t^{\prime}$. So the time between ticks actually seen on the clock is different from that given by the relativistic time dilation, just as the physical appearance of objects is different than that given by the Lorentz contraction.

\section{REMOVAL OF OPTICAL EFFECTS}

In this section we derive the transformation that removes the optical effects from a two-dimensional projection of an object, thereby leaving only the relativistic effects. Consider a point $(x(t), y, z)$ on the object at time $t$ (see Fig. 1). In Fig. 1 , the $z$-axis and $\zeta$-axis are directed out of the paper. Because light takes a finite time to reach the observer at $(0,0,0)$, the light from this point at time $t$ appears to come from $\left(x_{a}, y, z\right)$, where $x_{a}=x(t-\Delta t)$ and $\Delta t$ is the time the light takes to reach the observer. To find how $x$ and $x_{a}$ are related, we note that the distance the light travels in going from $\left(x_{a}, y, z\right)$ to $(0,0,0)$ is $c \Delta t=\sqrt{x_{a}^{2}+y^{2}+z^{2}}$. We also have that $x=x_{a}+v \Delta t$, where $v$ is the speed of the object. We combine these two equations to obtain

$$
x=x_{a}+\beta \sqrt{x_{a}^{2}+y^{2}+z^{2}},
$$

where $\beta=v / c$. Notice that the optical effects are first order in $\beta$, in contrast to the relativistic effects which are second order in $\beta$, because $\gamma=1 / \sqrt{1-\beta^{2}}$.

We assume that the projection plane for the actual coordinates of the object is such that it is perpendicular to a line segment from the observer to some reference point $\left(x_{0}, y_{0}, 0\right)$ of the object and a distance $d_{p}$ from the observer. The natural reference point would be the center of the object, although another point could be taken. We take $(\chi, \zeta)$ to be the coordinates on this projection plane, with $\zeta$ in the same direction as $z$. The coordinates $(\chi, \zeta)$ correspond to the intersection of the projection plane and the line segment from the observer to the point $(x, y, z)$; the origin $(0,0)$ of these coordinates corresponds to the intersection of the projection plane and the line segment from the observer to the reference point. Similarly, we take the projection plane for the apparent coordinates to be such that it is perpendicular to a line from the observer to the apparent reference point $\left(x_{0 a}, y_{0}, 0\right)$ and also a distance $d_{p}$ from the observer. The coordinates on this projection plane are taken to be $\left(\chi_{a}, \zeta_{a}\right)$.

Consider first the projection plane corresponding to the actual position of the object. To derive the coordinates $(\chi, \zeta)$ in terms of $x, y$, and $z$, it is simplest to first define a coordinate system $\left(x^{\prime \prime}, y^{\prime \prime}, z\right)$ that is rotated by an angle $\theta_{0}$ about the $z$-axis, so that the $y^{\prime \prime}$-axis is perpendicular to the projection plane. Then $\chi$ and $\zeta$ in terms of $x^{\prime \prime}, y^{\prime \prime}$, and $z$ are simply $\chi=d_{p} x^{\prime \prime} / y^{\prime \prime}$ and $\zeta=d_{p} z / y^{\prime \prime}$. If we rotate the coordinate system about the $z$ axis by $\theta_{0}$ using $x^{\prime \prime}=x \cos \theta_{0}-y \sin \theta_{0}$ and $y^{\prime \prime}=y \cos \theta_{0}+x \sin \theta_{0}$, we find

$$
\begin{aligned}
& \chi=\frac{d_{p}\left(x \cos \theta_{0}-y \sin \theta_{0}\right)}{y \cos \theta_{0}+x \sin \theta_{0}}, \\
& \zeta=\frac{d_{p} z}{y \cos \theta_{0}+x \sin \theta_{0}} .
\end{aligned}
$$

Similarly, we have for the apparent coordinates

$$
\begin{aligned}
& \chi_{a}=\frac{d_{p}\left(x_{a} \cos \theta_{0 a}-y \sin \theta_{0 a}\right)}{y \cos \theta_{0 a}+x_{a} \sin \theta_{0 a}}, \\
& \zeta_{a}=\frac{d_{p} z}{y \cos \theta_{0 a}+x_{a} \sin \theta_{0 a}} .
\end{aligned}
$$

The relation between $\theta_{0}$ and $\theta_{0 a}$ may be found by taking $x$ $=x_{0}, x_{a}=x_{0 a}, y=y_{0}$, and $z=0$ (that is, the reference point) and dividing both sides of Eq. (1) by $y_{0}$ giving

$$
\tan \theta_{0}=\tan \theta_{0 a}+\beta \sec \theta_{0 a} .
$$

We substitute Eq. (1) into Eq. (2) and obtain 
$\chi=d_{p} \frac{\left[\left(x_{a} / y\right)+\beta \sqrt{\left(x_{a} / y\right)^{2}+1+(z / y)^{2}}\right] \cos \theta_{0}-\sin \theta_{0}}{\cos \theta_{0}+\left[\left(x_{a} / y\right)+\beta \sqrt{\left(x_{a} / y\right)^{2}+1+(z / y)^{2}}\right] \sin \theta_{0}}$,

$$
\zeta=\frac{d_{p}(z / y)}{\cos \theta_{0}+\left[\left(x_{a} / y\right)+\beta \sqrt{\left(x_{a} / y\right)^{2}+1+(z / y)^{2}}\right] \sin \theta_{0}} .
$$

If we solve for $x_{a} / y$ and $z / y$ in Eq. (3), we obtain

$$
x_{a} / y=\frac{d_{p} \sin \theta_{0 a}+\chi_{a} \cos \theta_{0 a}}{d_{p} \cos \theta_{0 a}-\chi_{a} \sin \theta_{0 a}}
$$

$$
z / y=\frac{\zeta_{a}}{d_{p} \cos \theta_{0 a}-\chi_{a} \sin \theta_{0 a}},
$$

and, from Eq. (4),

$$
\begin{aligned}
& \sin \theta_{0}=\frac{\sin \theta_{0 a}+\beta}{\sqrt{1+2 \beta \sin \theta_{0 a}+\beta^{2}}}, \\
& \cos \theta_{0}=\frac{\cos \theta_{0 a}}{\sqrt{1+2 \beta \sin \theta_{0 a}+\beta^{2}}} .
\end{aligned}
$$

Although Eqs. (5)-(7) could be used directly, substantial simplification results if the equations are combined giving

$$
\begin{aligned}
& \chi=\frac{d_{p}\left[\chi_{a}\left(1+\beta \sin \theta_{0 a}\right)+\beta \cos \theta_{0 a}\left(\sqrt{d_{p}^{2}+\chi_{a}^{2}+\zeta_{a}^{2}}-d_{p}\right)\right]}{d_{p}\left(1+\beta \sin \theta_{0 a}\right)+\beta \chi_{a} \cos \theta_{0 a}+\beta\left(\sin \theta_{0 a}+\beta\right) \sqrt{d_{p}^{2}+\chi_{a}^{2}+\zeta_{a}^{2}}}, \\
& \zeta=\frac{d_{p} \zeta_{a} \sqrt{1+2 \beta \sin \theta_{0 a}+\beta^{2}}}{d_{p}\left(1+\beta \sin \theta_{0 a}\right)+\beta \chi_{a} \cos \theta_{0 a}+\beta\left(\sin \theta_{0 a}+\beta\right) \sqrt{d_{p}^{2}+\chi_{a}^{2}+\zeta_{a}^{2}}} .
\end{aligned}
$$

Equation (8) gives the actual two-dimensional projection coordinates $(\chi, \zeta)$ in terms of the apparent two-dimensional projection coordinates $\left(\chi_{a}, \zeta_{a}\right)$. The actual angle $\theta_{0}$ in terms of the apparent angle $\theta_{0 a}$ is given by Eq. (4). As discussed, Eq. (8) removes the optical effects, leaving only the relativistic effects. These equations will be discussed further toward the end of Sec. IV.

Although some of the complication seen in Eq. (8) results from the fact that a three-dimensional object is projected onto a $2 \mathrm{D}$ plane, most is related to the projection plane being directed so that the projection of the reference point lies at the center of the projection plane for both the actual and apparent positions of the object. If the image is instead projected onto a sphere, the transformation between the apparent angles $\left(\theta_{a}, \phi_{a}\right)$ and actual angles $(\theta, \phi)$ on the sphere is given by

$$
\tan \theta=\tan \theta_{a}+\beta \sec \theta_{a}, \quad \phi=\phi_{a} .
$$

Here $\phi$ is the azimuthal angle and $\theta$ is an angle measured from the "equator." If we instead use the usual polar angle, $\theta$ would need to be replaced by $\pi / 2-\theta$, giving $\cot \theta=\cot \theta_{a}$ $+\beta \csc \theta_{a}$.

\section{THE APPARENT SPEED}

Because it takes a finite time for light to travel from the object to the observer, the object will appear to be behind its actual position, which will result in the apparent speed being different from the actual speed. If we solve for $x_{a}$ in Eq. (1), we find

$$
x_{a}=\frac{x-\beta \sqrt{x^{2}+\left(1-\beta^{2}\right)\left(y^{2}+z^{2}\right)}}{1-\beta^{2}} .
$$

For simplicity, we assume that the object is far away so that it looks like a point. Then we have $z=0$ and $y=d_{c}$, where $d_{c}$ is the distance of closest approach. We note that $x=v t$ and divide both sides of Eq. (10) by $d_{c}$ to find the dimen- sionless apparent displacement and the tangent of the apparent angle

$$
\frac{x_{a}}{d_{c}}=\tan \theta_{a}=\frac{\tau-\beta \sqrt{\tau^{2}+\left(1-\beta^{2}\right)}}{1-\beta^{2}},
$$

where $\tau=v t / d_{c}$ is the dimensionless time. Figure 2 shows a plot of $\tan \theta_{a}$ as a function of $\tau$ for various values of $\beta$. The slope of this plot gives the dimensionless apparent speed $v_{a} / v$. If we take the derivative of Eq. (11) with respect to $\tau$, we obtain

$$
\frac{v_{a}}{v}=\frac{1-\beta \tau\left(\tau^{2}+1-\beta^{2}\right)^{-1 / 2}}{1-\beta^{2}} .
$$

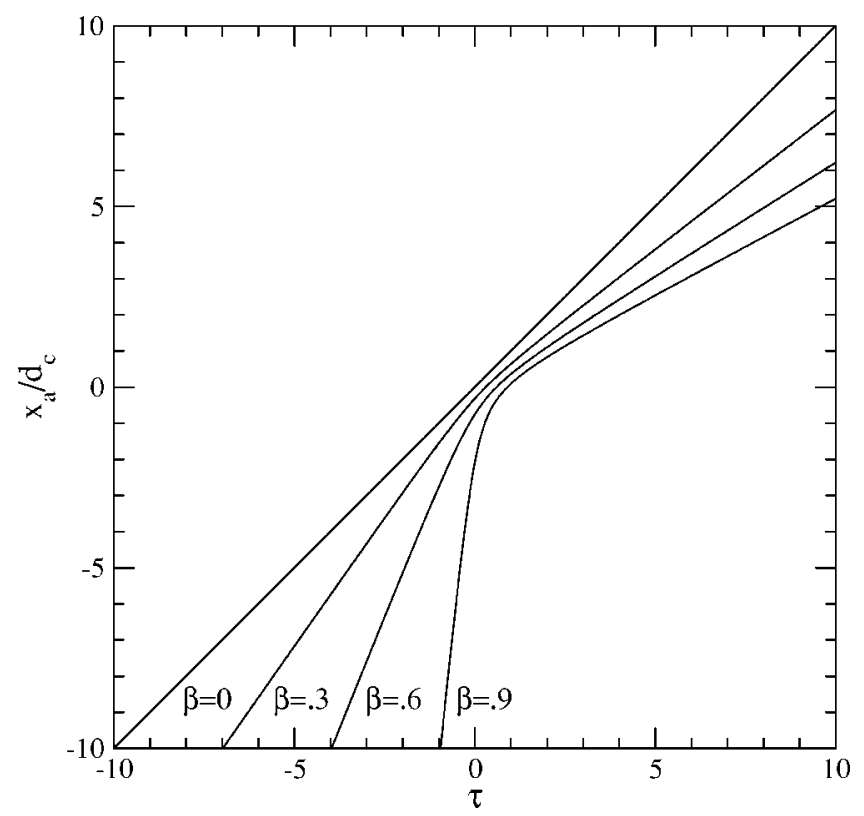

Fig. 2. Apparent (dimensionless) position of an object as a function of the dimensionless time for various values of $\beta=v / c$. 
Figure 3 shows a plot of $v_{a} / v$ as a function of $\tau$. Note that as the object is approaching, the apparent speed is larger than $v$ and as the object is receding, the apparent speed is smaller than $v$. As expected, the apparent speed deviates more from the actual speed for larger values of $\beta$. In the limit of large $|\gamma|$ we have

$$
\lim _{\tau \rightarrow \pm \infty} v_{a} / v=\frac{1}{1 \pm \beta}
$$

Note that as $\beta \rightarrow 1$, the apparent speed approaches $\infty$ as the object approaches, and the apparent speed approaches $\frac{1}{2}$ that of the actual speed as the object recedes. Therefore, the apparent speed of the object can be much greater than the speed of light for an approaching object.

If an object is moving past at a relativistic speed, we can plot the angle at which the object is seen, that is, the apparent angle, as a function of time and then fit the curve to a function of the form

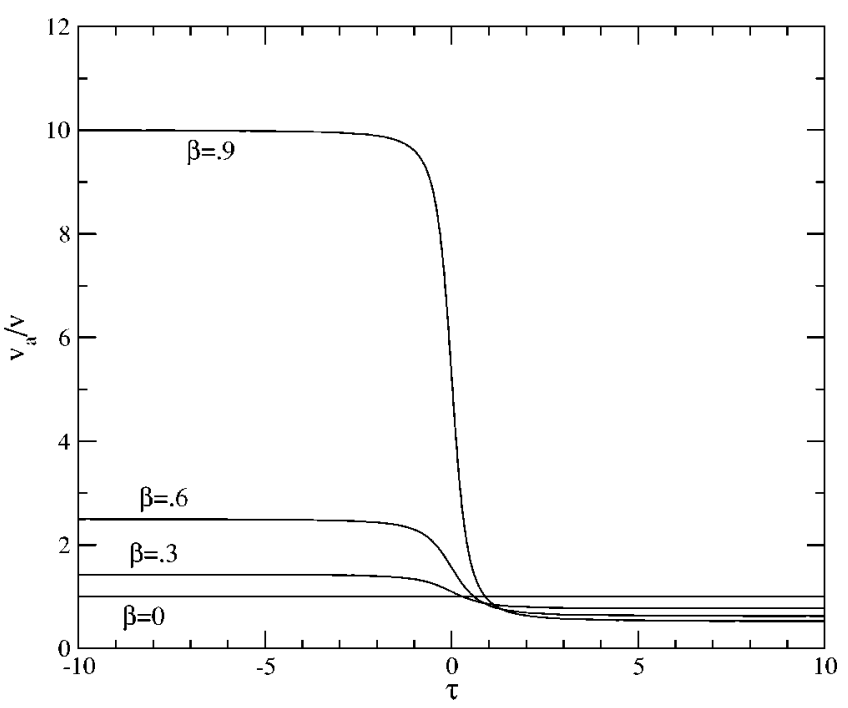

Fig. 3. Apparent (dimensionless) speed of an object as a function of the dimensionless time for various values of $\beta$.

$$
\theta_{a}=\theta_{a c}+\tan ^{-1}\left[\frac{\alpha\left(t-t_{c}+\beta / \alpha\right)-\beta \sqrt{\alpha^{2}\left(t-t_{c}+\beta / \alpha\right)^{2}+1-\beta^{2}}}{1-\beta^{2}}\right]
$$

where $\alpha=v / d_{c}$. The time was shifted by $\beta / \alpha$ so that when $t=t_{c}, \theta_{a}=\theta_{a c}$, the angle of closest approach. Given $\theta$ as a function of $t$, we can fit the curve by varying the parameters $\theta_{a c}, t_{c}, \alpha$, and $\beta$. The parameters $\theta_{a c}$ and $t_{c}$ will move the curve vertically and horizontally, respectively, and give the angle and time of closest approach. If the object is moving non-relativistically $(\beta=0)$, in addition to $\theta_{a c}$ and $t_{c}$, only the parameter $\alpha$ could be determined and thus only the ratio of $v$ and $d_{c}$ could be determined. However, if the object is moving relativistically, both $\alpha$ and $\beta$ can be determined and therefore both $v$ and $d_{c}$ could be determined, which is in sharp contrast to the nonrelativistic case.

\section{THE APPEARANCE OF RELATIVISTIC OBJECTS}

As noted, there has been considerable work on the appearance of objects moving at relativistic speeds. ${ }^{2-17}$ However, the equations that transform the two-dimensional projection coordinates of an object in its rest frame directly to the apparent two-dimensional projection coordinates of the object have not been derived. We derive these equations and apply them to photographic images, demonstrating what the objects would look like if they were moving at relativistic speeds.

Consider an object moving in the positive $x$-direction as in Fig. 1. In addition to the $(x, y, z)$ frame of reference shown in the figure, also consider a frame of reference $\left(x^{\prime}, y^{\prime}, z^{\prime}\right)$ in which the object is at rest. Because $y^{\prime}=y$ and $z^{\prime}=z$, we will drop the primes for these variables. The relation between $x$ and $x^{\prime}$ is given by $x=(1 / \gamma) x^{\prime}+v t$. At the instant the origins coincide, two observers $O$ and $O^{\prime}$ at the origins of their respective reference frames compare measurements, giving $x=(1 / \gamma) x^{\prime}$. If we substitute this relation into Eq. (10), we obtain

$$
x_{a}=\gamma x^{\prime}-\gamma \beta \sqrt{x^{\prime 2}+y^{2}+z^{2}} .
$$

Before deriving the transformation equations for the twodimensional projection of the object, we derive a few relations that quantify some of the changes in the object's appearance. Only when the object appears to be directly in front of the observer is the apparent length of the object equal to the quantity $1 / \gamma$ times the rest length of the object. ${ }^{8}$ As the object appears to be approaching, the length appears longer than this quantity and when the object appears to be receding, the length appears shorter than this quantity. ${ }^{5}$ To quantify this length change, we calculate $\partial x_{a} / \partial x^{\prime}$ from Eq. (15) and evaluate the derivative at $\left(x^{\prime}, y, z\right)=\left(x_{0}^{\prime}, d_{c}, 0\right)$, giving

$$
\partial x_{a} / \partial x^{\prime}=\gamma-\frac{\beta \gamma\left(x_{0}^{\prime} / d_{c}\right)}{\sqrt{\left(x_{0}^{\prime} / d_{c}\right)^{2}+1}} .
$$

Figure 4 shows a plot of $\partial x_{a} / \partial x^{\prime}$. Note that if one is subtracted from the derivative, it is in the form of a strain, that is, $\partial\left(x_{a}-x^{\prime}\right) / \partial x^{\prime}$. As we have mentioned, the length of the object appears shortened by a factor of $1 / \gamma$ when $x_{0 a}=0$. From Eq. (15), we see that when $x_{0 a}=0, x_{0}^{\prime}=\gamma \beta d_{c}$. The substitution of this value for $x_{0}^{\prime}$ into Eq. (16) gives $\partial x_{a} / \partial x^{\prime}=1 / \gamma$ as expected. Also, when $x_{0 a}<0$, that is, $x_{0}^{\prime}$ $<\gamma \beta d_{c}$, the apparent length is larger than $1 / \gamma$ and when $x_{0 a}>0$, that is, $x_{0}^{\prime}>\gamma \beta d_{c}$, the apparent length is smaller than $1 / \gamma$.

Because light from points on the object that are further from the observer take a longer time to reach the observer, the object will appear to be sheared as compared to the ob- 


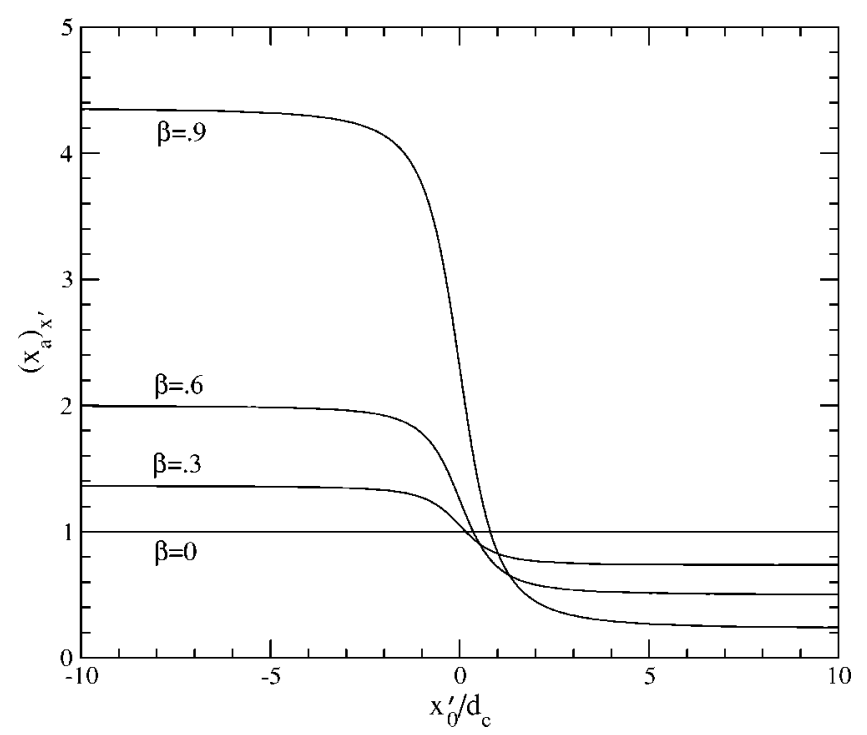

Fig. 4. Factor by which the length is changed, $\partial x_{a} / \partial x^{\prime}$, as a function of the dimensionless rest-frame position.

ject at rest, with the back of the object behind the front of the object (see Fig. 1). ${ }^{5,8}$ To quantify this shear we calculate $\partial x_{a} / \partial y$ from Eq. (15) and evaluate it at $\left(x^{\prime}, y, z\right)$ $=\left(x_{0}^{\prime}, d_{c}, 0\right)$, giving

$$
\frac{\partial x_{a}}{\partial y}=-\frac{\beta \gamma}{\sqrt{\left(x_{0}^{\prime} / d_{c}\right)^{2}+1}} .
$$

If the object is large, that is, the size of the object cannot be neglected relative to the distance of the object from the observer, the object also will appear to be curved in the $z$-direction in comparison to the object at rest. That is, for a given value of $y$, points higher and lower on the object will appear behind points closer to the center. ${ }^{5,8}$ To quantify this curvature we calculate $d_{c}\left(\partial^{2} x_{a} / \partial z^{2}\right)$ from Eq. (15) and evaluate it at $\left(x^{\prime}, y, z\right)=\left(x_{0}^{\prime}, d_{c}, 0\right)$, giving

$$
d_{c} \frac{\partial^{2} x_{a}}{\partial z^{2}}=-\frac{\beta \gamma}{\sqrt{\left(x_{0}^{\prime} / d_{c}\right)^{2}+1}} .
$$

Because both the shear and curvature as defined in Eqs. (17) and (18), respectively, are the same function, Fig. 5 shows both $\partial x_{a} / \partial y$ and $d_{c}\left(\partial^{2} x_{a} / \partial z^{2}\right)$. We note that $\partial x_{a} / \partial z$ is zero about the point $\left(x^{\prime}, y, z\right)=\left(x_{0}^{\prime}, d_{c}, 0\right)$. If $z_{0}$ were not zero, then this quantity would not be zero. In fact, $\partial x_{a} / \partial x^{\prime}$, $\partial x_{a} / \partial y$, and $\partial x_{a} / \partial z$ can be calculated at any point to give the apparent deformation at any point. The quantities $d_{c}\left(\partial^{2} x_{a} / \partial x^{\prime 2}\right)$ and $d_{c}\left(\partial^{2} x_{a} / \partial y^{2}\right)$ also can be calculated.

We now derive the transformation equations for the twodimensional projection of the object. Just as we had done for the apparent coordinates and actual coordinates in Sec. II, we take the projection plane for the primed coordinates to be such that it is perpendicular to a line from the observer to the reference point $\left(x_{0}^{\prime}, y_{0}, 0\right)$ and also a distance $d_{p}$ from the observer. We take the coordinates on this projection plane as $\left(\chi^{\prime}, \zeta^{\prime}\right)$. If we take the primed coordinates in Eq. (15) to be the reference point $\left(x_{0}^{\prime}, y_{0}, 0\right)$ and divide both sides by $y_{0}$, we find

$$
\tan \theta_{0 a}=\gamma \tan \theta_{0}^{\prime}-\gamma \beta \sec \theta_{0}^{\prime} .
$$

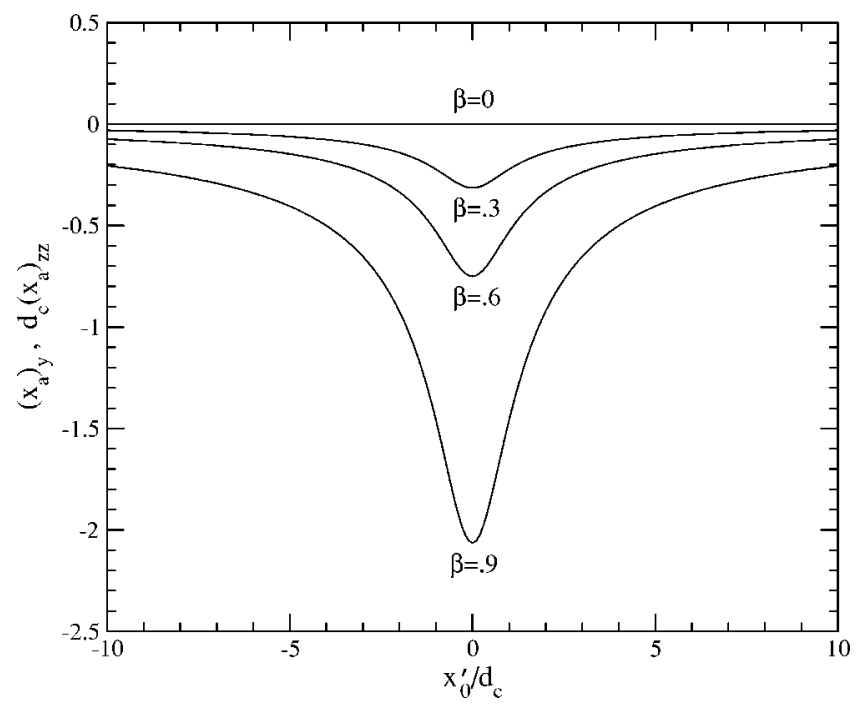

Fig. 5. The shear, $\partial x_{a} / \partial y$, and curvature, $d_{c}\left(\partial^{2} x_{a} / \partial z^{2}\right)$, as a function of the dimensionless rest-frame position.

Similar to Eqs. (2) and (3) we have for the primed projection coordinates

$$
\begin{aligned}
& \chi^{\prime}=\frac{d_{p}\left(x^{\prime} \cos \theta_{0}^{\prime}-y^{\prime} \sin \theta_{0}^{\prime}\right)}{y^{\prime} \cos \theta_{0}^{\prime}+x^{\prime} \sin \theta_{0}^{\prime}}, \\
& \zeta^{\prime}=\frac{d_{p} z^{\prime}}{y^{\prime} \cos \theta_{0}^{\prime}+x^{\prime} \sin \theta_{0}^{\prime}} .
\end{aligned}
$$

The substitution of Eq. (15) into Eq. (3) gives

$\chi_{a}=d_{p} \frac{\gamma\left[\left(x^{\prime} / y\right)-\beta \sqrt{\left(x^{\prime} / y\right)^{2}+1+(z / y)^{2}}\right] \cos \theta_{0 a}-\sin \theta_{0 a}}{\cos \theta_{0 a}+\gamma\left[\left(x^{\prime} / y\right)-\beta \sqrt{\left(x^{\prime} / y\right)^{2}+1+(z / y)^{2}}\right] \sin \theta_{0 a}}$,

$\zeta_{a}=\frac{d_{p}(z / y)}{\cos \theta_{0 a}+\gamma\left[\left(x^{\prime} / y\right)-\beta \sqrt{\left(x^{\prime} / y\right)^{2}+1+(z / y)^{2}}\right] \sin \theta_{0 a}}$,

where, solving for $x^{\prime} / y$ and $z / y$ in Eq. (20),

$$
\begin{aligned}
& \frac{x^{\prime}}{y}=\frac{d_{p} \sin \theta_{0}^{\prime}+\chi^{\prime} \cos \theta_{0}^{\prime}}{d_{p} \cos \theta_{0}^{\prime}-\chi^{\prime} \sin \theta_{0}^{\prime}}, \\
& \frac{z}{y}=\frac{\zeta^{\prime}}{d_{p} \cos \theta_{0 a}-\chi^{\prime} \sin \theta_{0 a}},
\end{aligned}
$$

and, from Eq. (19),

$$
\begin{aligned}
& \sin \theta_{0 a}=\frac{\sin \theta_{0}^{\prime}-\beta}{1-\beta \sin \theta_{0}^{\prime}}, \\
& \cos \theta_{0 a}=\frac{\cos \theta_{0}^{\prime}}{\gamma\left(1-\beta \sin \theta_{0}^{\prime}\right)} .
\end{aligned}
$$

We combine Eqs. (21)-(23) to find 


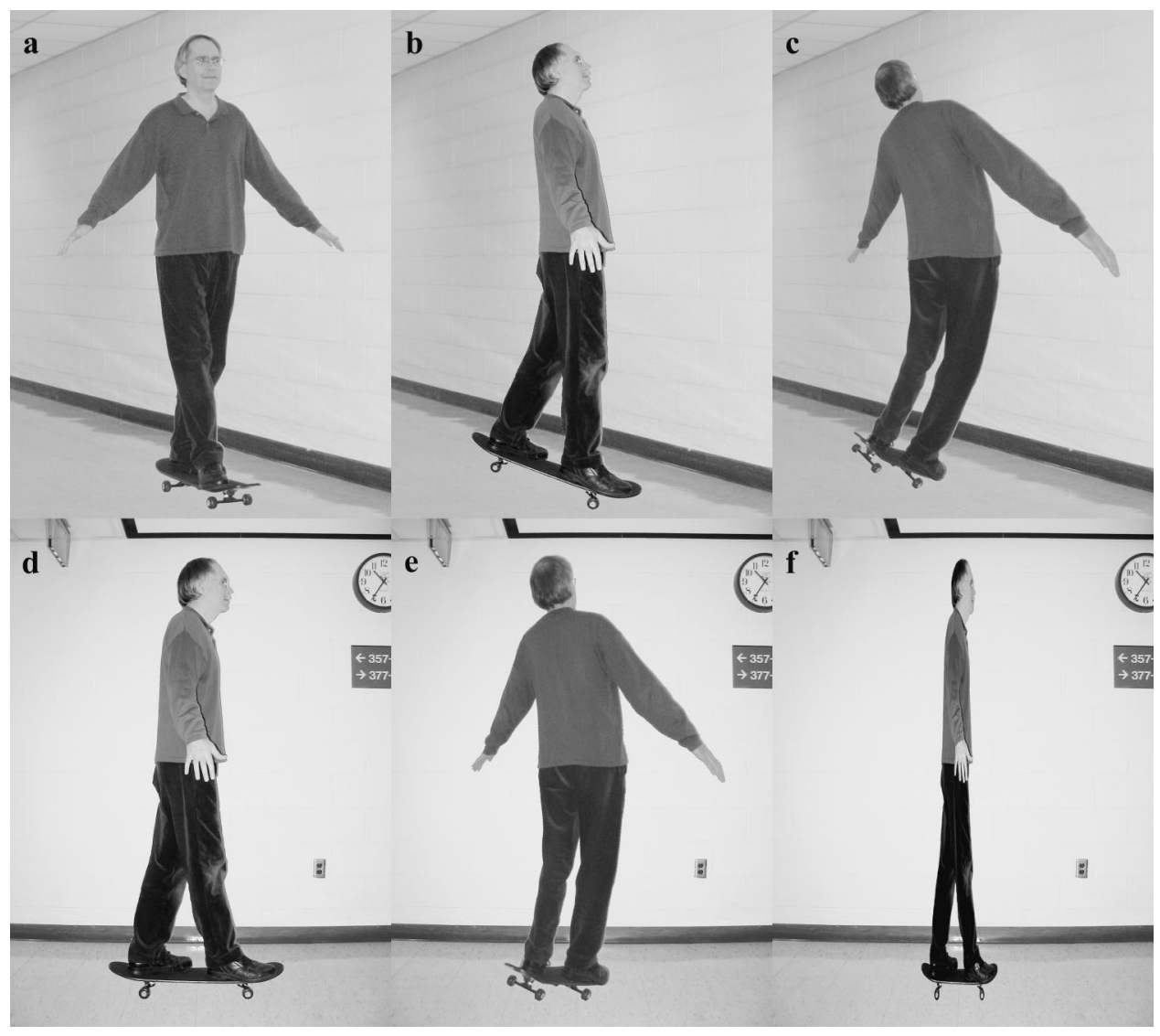

Fig. 6. The author on a skateboard moving down a hall at various apparent angles $\theta_{0 a}$ and various values of $\beta$. (a) $\theta_{0 a}=-64.15^{\circ}, \quad \beta=0$, (b) $\theta_{0 a}$ $=-64.15^{\circ}, \quad \beta=0.9, \quad$ (c) $\theta_{0 a}$ $=-64.15^{\circ}, \beta=0.9945$, (d) $\theta_{0 a}=0^{\circ}$, $\beta=0$, (e) $\theta_{0 a}=0^{\circ}, \beta=0.9$, and (f) $\theta_{0}=0^{\circ}, \beta=0.9$, showing only the relativistic effects.

$$
\begin{aligned}
& \chi_{a}=\frac{d_{p}\left[\chi^{\prime}\left(1-\beta \sin \theta_{0}^{\prime}\right)-\beta \cos \theta_{0}^{\prime}\left(\sqrt{d_{p}^{2}+\chi^{\prime 2}+\zeta^{\prime 2}}-d_{p}\right)\right]}{\gamma\left[d_{p}\left(1-\beta \sin \theta_{0}^{\prime}\right)^{2}-\chi^{\prime} \cos \theta_{0}^{\prime} \beta\left(1-\beta \sin \theta_{0}^{\prime}\right)-\beta\left(\sin \theta_{0}^{\prime}-\beta\right)\left(\sqrt{d_{p}^{2}+\chi^{\prime 2}+\zeta^{\prime 2}}-d_{p}\right)\right]}, \\
& \zeta_{a}=\frac{d_{p} \zeta^{\prime}\left(1-\beta \sin \theta_{0}^{\prime}\right)}{\gamma\left[d_{p}\left(1-\beta \sin \theta_{0}^{\prime}\right)^{2}-\chi^{\prime} \cos \theta_{0}^{\prime} \beta\left(1-\beta \sin \theta_{0}^{\prime}\right)-\beta\left(\sin \theta_{0}^{\prime}-\beta\right)\left(\sqrt{d_{p}^{2}+\chi^{\prime 2}+\zeta^{\prime 2}}-d_{p}\right)\right]} .
\end{aligned}
$$

Equation (24) gives the apparent two-dimensional projection coordinates $\left(\chi_{a}, \zeta_{a}\right)$ in terms of the rest-frame twodimensional projection coordinates $\left(\chi^{\prime}, \zeta^{\prime}\right)$. The apparent angle in terms of the rest-frame angle is given by Eq. (19). In the limit of a small subtended solid angle, $\chi^{\prime} / d_{p} \ll 1$ and $\zeta^{\prime} / d_{p} \ll 1$, Eq. (24) reduces to the results of Terrell, ${ }^{3}$

$$
\begin{aligned}
& \chi_{a}=\frac{\chi^{\prime}}{\gamma\left(1-\beta \sin \theta_{0}^{\prime}\right)}, \\
& \zeta_{a}=\frac{\zeta^{\prime}}{\gamma\left(1-\beta \sin \theta_{0}^{\prime}\right)} .
\end{aligned}
$$

Because both $\chi^{\prime}$ and $\zeta^{\prime}$ are multiplied by the same constant, the apparent shape is the same as the rest-frame shape. Because the apparent shape is observed from a different angle than the rest-frame shape, as given by Eq. (19), and the coefficient $1 /\left[\gamma\left(1-\beta \sin \theta_{0}^{\prime}\right)\right]$ is equal to the ratio of the distance between the observer and the object in the rest frame to the distance between the observer and the apparent position of the object, the object will simply look as if it has been rotated by the angle $\theta_{0 a}-\theta_{0}^{\prime}$, in comparison to an identical stationary object located at the apparent position of the ob- ject. This apparent rotation is the basis of the Terrell effect, that is, an object moving at a relativistic speed will look as if it has been rotated. Although the appearance is that of being rotated, it is more accurate to state that the object appears sheared and changed in length, which becomes clear if the object is, for example, a car on a straight road or a train car on a straight track.

Again the complication in Eq. (24) is for the same reasons as discussed after Eq. (8). If, instead of being projected onto a plane, the image is projected onto a sphere, the transformation between the rest-frame and apparent angles on the sphere is given by

$$
\tan \theta_{a}=\gamma \tan \theta^{\prime}-\gamma \beta \sec \theta^{\prime}, \quad \phi_{a}=\phi^{\prime} .
$$

Just as for Eq. (9), $\phi$ is the azimuthal angle and $\theta$ is an angle measured from the "equator." If we instead use the usual polar angle, $\theta$ would be replaced by $\pi / 2-\theta$. Then the equation for $\theta$ would be in the form of the well-known relativistic aberration equation, ${ }^{2,3,15,16}$ that is, $\cot \theta_{a}=\gamma \cot \theta^{\prime}$ $-\gamma \beta \csc \theta^{\prime}$.

Figure 6 shows the results of applying Eq. (24) to the positions of the pixels for some photographic images. To obtain these images, the image that will appear to be moving 
is photographed from different angles against a solid color background (to make extracting the image from the background easier). The angles are determined from Eq. (19). For example, if the apparent position of the image is directly in front of the camera (that is, $\theta_{0 a}=0^{\circ}$ ) and the image is moving at a velocity of $\beta=0.9$, then the image that is photographed would be moving away at the angle $\theta_{0}^{\prime}=64.15^{\circ}$ as given by Eq. (19). Also the distance that the camera is placed from the image is $d_{c} \sec \left(\theta_{0}^{\prime}\right)$, where $d_{c}$ is the distance of closest approach ( $d_{c} \approx 94^{\prime \prime}$ for Fig. 6). An optical zoom is used on those images that are far away, that is, moving toward or away from the camera, to eliminate the loss of digital resolution. For example, when the image is photographed at an angle of $\theta_{0}^{\prime}=64.15^{\circ}$, a magnification of $\approx 2.5$ was used. Equation (24) is then applied to the images that are moving at relativistic speeds. For the images for which the optical zoom was used, the image needs to be reduced by the same factor before applying the transformation. Pictures of the hallway were taken at two angles, $-64.15^{\circ}$ and $0^{\circ}$. The moving images were than superimposed on the hallway. The computer program ${ }^{19}$ was written in Java.

Figures 6(a)-6(c) show the image moving toward the observer at an angle of $-64.15^{\circ}$ for $\beta=0, \beta=0.9$, and $\beta$ $=0.9945$, respectively. Figures 6(d) and 6(e) show the image when it appears to be directly in front of the observer for $\beta=0$ and $\beta=0.9$. The speed of $\beta=0.9945$ was determined by taking $\theta_{0 a}=-\theta_{0}^{\prime}$ in Eq. (19) and solving for $\beta$,

$$
\beta=\frac{2 \tan \theta_{0}^{\prime} \sec \theta_{0}^{\prime}}{1+2 \tan ^{2} \theta_{0}^{\prime}}
$$

with $\theta_{0}^{\prime}=64.15^{\circ}$. We were therefore able to use the same photo that was used to obtain the moving image in Fig. 6(e), as well as the same background used in Figs. 6(a) and 6(b).

If we compare the figures for which the image is moving relativistically [Figs. 6(b), 6(c), and 6(e)] to those for which the image is moving nonrelativistically $[\beta=0$, Figs. $6(\mathrm{a})$ and $6(d)]$, we see that the dominant effect is that of appearing to be rotated. The moving images in Figs. 6(b) and 6(e) appear rotated by an angle of $64.15^{\circ}$ in comparison to the moving images in Figs. 6(a) and 6(d), respectively; the moving image in Fig. 6(c) appears rotated by an angle of $128.3^{\circ} \mathrm{com}-$ pared to the moving image in Fig. 6(a). The curvature discussed near the beginning of this section [see Eq. (18)] is seen in Figs. 6(b), 6(c), and 6(e). Also notice how the skateboard points directly down the hallway for all the relativistically moving images [Figs. 6(b), 6(c), and 6(e)], which again reinforces the point that it is more accurate to state that the image appears sheared and changed in length, rather than rotated, because if the image were simply rotated, the skateboard would not point directly down the hallway.

Finally, Fig. 6(f) shows the result if the relativistically moving image simply appeared contracted by a factor of $1 / \gamma$. Figure 6(f) also would be the result if the optical effects were removed by applying Eq. (8) to the moving image of Fig. 6(b), leaving only the relativistic effects, as discussed in Sec. II. If we compare Figs. 6(e) and 6(f), we see that what we see and how an object appears are indeed very different from what is given by the Lorentz contraction.

${ }^{a)}$ For current e-mail address and other contact information < http://
www.deissler.us $>$.

${ }^{1}$ A. Lampa, "Wie erscheint nach der Relativitaetstheorie ein bewegter Stab einem ruhenden Beobachter?" ("How does a moving rod appear for an observer at rest according to the theory of relativity?"), Z. Phys. 27, 138148 (1924)

${ }^{2}$ R. Penrose, "The apparent shape of a relativistically moving sphere," Proc. Cambridge Philos. Soc. 55, 137-139 (1959).

${ }^{3}$ J. Terrell, "The invisibility of the Lorentz contraction," Phys. Rev. 116, 1041-1045 (1959).

${ }^{4}$ V. F. Weisskopf, “The visual appearance of rapidly moving objects," Phys. Today 13(9), 24-27 (1960).

${ }^{5}$ F. R. Hickey, "Two-dimensional appearance of a relativistic cube," Am. J. Phys. 47, 711-714 (1979).

${ }^{6}$ D. W. Lang, "The meter stick in a match box," Am. J. Phys. 38, 11811184 (1970).

${ }^{7}$ P. M. Matthews and M. Lakshmanan, "On the apparent visual forms of relativistically moving objects," Nuovo Cimento 12, 168-180 (1972).

${ }^{8}$ G. D. Scott and M. R. Viner, "The geometrical appearance of large objects moving at relativistic speeds," Am. J. Phys. 33, 534-537 (1965).

${ }^{9}$ G. D. Scott and H. J. van Driel, "Geometrical appearances at relativistic speeds," Am. J. Phys. 38, 971-977 (1970).

${ }^{10}$ K. G. Suffern, "The apparent shape of a rapidly moving sphere," Am. J. Phys. 56, 729-733 (1988).

${ }^{11}$ D. Hollenbach, "Appearance of a rapidly moving sphere: A problem for undergraduates,” Am. J. Phys. 44, 91-93 (1976).

${ }^{12}$ J. R. Burke and F. J. Strode, "Classroom exercises with the Terrell effect," Am. J. Phys. 59, 912-915 (1991)

${ }^{13}$ P.-K. Hsiung and R. H. P. Dunn, "Visualizing relativistic effects in spacetime," in Proceedings of Supercomputing '89 Conference (ACM, 1989), pp. 597-606

${ }^{14}$ P.-K. Hsiung, R. H. Thibadeau, and M. Wu, “T-buffer: Fast visualization of relativistic effects in spacetime," Comput. Graphics 24(2), 83-88 (1990).

${ }^{15}$ D. Weiskopf, "A texture mapping approach for the visualization of special relativity," in IEEE Visualization 1999 Late Breaking Hot Topics Proceedings, edited by A. Varshney, C. M. Wittenbrink, and H. Hagen (ACM, 1999), pp. 41-44, or 〈http://www.tat.physik.unituebingen.de/weiskopf/publications/vis99.pdf).

${ }^{16}$ D. Weiskopf, D. Kobras, and H. Ruder, "Real-world relativity: Imagebased special relativistic visualization," in IEEE Visualization 2000 Proceedings, edited by T. Ertl, B. Hamann, and A. Varshney (ACM, 2000), pp. 303-310, or 〈http://wwwvis.informatik.unistuttgart.de/weiskopf/publications/vis00ibr.pdf).

${ }^{17}$ U. Kraus, H. Ruder, D. Weiskopf, and C. Zahn, "Was Einstein noch nicht sehen konnte. Schnelle Computer visualisieren relativistische Effekte," Phys. J. 7/8, 77-83 (2002) or 〈http://www.tempolimitlichtgeschwindigkeit.de/tompkins/tompkinsHTML/ index.html).

${ }^{18}$ L. W. Epstein, Relativity Visualized (Insight, San Francisco, 1985).

${ }^{19}$ R. J. Deissler, "A Java program for transforming a photographic image to show the image moving at a relativistic speed" 〈http:// www. deissler.us $\rangle$.

${ }^{20}$ P.-K. Hsiung, R. H. Thibadeau, C. B. Cox, and R. H. P. Dunn, "Time dilation visualization in relativity," in Proceedings of the Supercomputing 1990 Conference (ACM, 1990), pp. 835-844. 